\title{
PURIFICAÇÃO DO BIODIESEL DE ÓLEO DE SOJA POR MEIO DO EMPREGO DE LÍQUIDOS IÔNICOS ANÁLOGOS BASEADOS EM CLORETO DE COLINA
}

\section{PURIFICATION OF SOYBEAN OIL-BASEAD BIODIESEL BY CHOLINE CHLORIDE-BASED IONIC LIQUIDS ANALOGOUS}

\author{
M. R. M. SANTOS ${ }^{1, *}$, E. A. SILVA ${ }^{1}$ E F. TAVARES ${ }^{1}$ \\ ${ }^{1}$ Universidade Estadual do Oeste do Paraná, Centro de Engenharias e Ciências Exatas, Toledo, Paraná, Brasil, \\ romeiro.matheus@hotmail.com \\ ${ }^{*}$ Corresponding author. Western Parana State University, Department of Engineering and Exact Sciences, Toledo, Parana, Brazil, Phone: $+55453379-7061$ \\ e-mail address: romeiro.matheus@hotmail.com (M.R.M. Santos).
}

\begin{tabular}{l} 
A R T I C L E I N F O \\
\hline Article history: \\
Received 2019-12-04 \\
Accepted 2020-06-17 \\
Available online 2020-06-17 \\
pa la vr a s - ch $a v e$ \\
Biodiesel \\
Líquido Iônico \\
Extração \\
Purificação \\
$k$ e ywor $d s$ \\
Biodiesel \\
Ionic liquid \\
Extraction \\
Purification
\end{tabular}

\begin{abstract}
A B S T R A C T
Traditional biodiesel purification processes are slow and costly, generating large volumes of effluents to meet the quality parameters required by legislation. This work evaluates the effects of new combinations for the synthesis of ionic liquids (LI's) on the efficiency of the solvent in removing impurities from soy biodiesel. Choline chloride-based LI's analogs, synthesized with the eutectic mixture of glycerol, ethylene glycol and triethylene glycol, were used in different ratios to wash pre-purified biodiesel. The IL L9 composed of choline chloride, glycerol and triethylene glycol (1:2:1), had the highest efficiency (98.05\%) when used in the ratio R2 (1:1), with an optimum washing time of 60 minutes. Crude biodiesel was also purified with the new solvent and showed good efficiency even when reused, in accordance with RANP 45/2014.
\end{abstract}

\section{R ES UMO}

Os processos tradicionais de purificação do biodiesel são onerosos e lentos, gerando grandes volumes de efluentes para atender os parâmetros de qualidade exigidos pela legislação. Este trabalho avalia os efeitos de novas combinações para a síntese de líquidos iônicos (LI's) sobre a eficiência do solvente na remoção de impurezas do biodiesel de soja. Os LI's análogos a base de cloreto de colina, sintetizados com a mistura eutética de glicerol, etilenoglicol e trietilenoglicol, foram usados para lavar o biodiesel pré-purificado em diferentes razões. $O$ LI L9 composto por cloreto de colina, glicerol e trietilenoglicol (1:2:1), teve a maior eficiência (98,05\%) quando usado na razão $R 2$ (1:1), com um tempo ótimo de lavagem de 60 minutos. O biodiesel bruto também foi purificado com o novo solvente e apresentou uma boa eficiência mesmo quando reutilizado, em conformidade com a RANP 45/2014. 


\section{INTRODUCÃO}

Os constantes avanços tecnológicos, que caracterizam a atual sociedade moderna, demandam de grandes quantidades de energia, sendo que cerca de $90 \%$ desta ainda é obtida de fontes não renováveis, como os combustíveis de origem fóssil (derivados do petróleo, gás natural e carvão mineral). A dependência mundial dos combustíveis fósseis causa preocupação, considerando estudos que indicam o esgotamento de suas fontes em algumas décadas e o risco ambiental que a queima deste material representa, devido a elevada emissão de poluentes atmosféricos $\left(\mathrm{CO}_{2}, \mathrm{SO}_{2}, \mathrm{NOx}\right)$, contribuindo para o aumento do efeito estufa (Bankovili ï Ilil et al., 2017; Kayode e Hart, 2019). Assim, inúmeros países têm adotado políticas de incentivo a diversificação de suas matrizes energéticas, por meio da inclusão de fontes de energia de origem renováveis, estimando que $50 \%$ da energia global até 2040, passará a ser obtida de maneira sustentável (Bankovilï Ilil et al., 2017; Martin et al., 2012).

Neste contexto, os biocombustíveis, que são derivados de biomassa renovável de origem vegetal e animal, se destacam pelo seu potencial, com viabilidade para substituir os combustíveis fósseis e superar os problemas ambientais relacionados a queima desse material não renovável. O biodiesel é um biocombustível amplamente utilizado no Brasil, produzido principalmente a partir de óleos vegetais (soja, semente de colza, semente de girassol, palma, farelo de arroz), sua queima representa uma redução considerável das emissões dos gases de efeito estufa, com baixos teores de enxofre e aromáticos (Ishak et al., 2017; Shahbaz et al., 2013). A alta eficiência de combustão do biodiesel, que possui um elevado ponto de fulgor e boas propriedades lubrificantes, permite seu emprego como combustível puro (B100), misturas com diesel de petróleo e ainda como aditivo (Bankovilï Iliĺ et al., 2017; Shahbaz et al., 2013).

Em escala industrial, o biodiesel geralmente é produzido por meio da reação de transesterificação, onde os triglicerídeos (TG) presentes no óleo vegetal, reagem com um álcool de cadeia curta, como o etanol e o metanol, na presença de um catalisador homogêneo (ácido ou básico), para formar glicerol e a mistura de ésteres monoalquílicos de ácidos graxos, conhecida como biodiesel (Ishak et al., 2017; Kayode e Hart, 2019; Taher et al., 2019). A transesterificação ocorre em três etapas consecutivas, produzindo uma molécula de éster por etapa, além de subprodutos, como diglicerídeos (DG), monoglicerídeos (MG) e glicerol (Kayode e Hart, 2019; Patel e Shah et al., 2015).

Ao final da reação de transesterificação ocorre a primeira etapa de purificação, na qual o biodiesel bruto, com alta concentração de ésteres monoalquílicos, é separado do glicerol de baixa solubilidade por meio de processos como centrifugação e a decantação por gravidade. Esta primeira separação ocorre de maneira espontânea devido a diferença considerável de densidade entre a fase do biodiesel bruto (å880 kg/m³) e do glicerol (å1050 kg/m³) (Atadashi et al., 2011; Ho et al., 2015; Kayode e Hart, 2019). bruto é submetido a uma etapa de lavagem com água quente para remover glicerol, glicerídeos, sabão, álcool, catalisador residual e outras impurezas dissolvidas na mistura de ésteres (Atadashi et al., 2011). A presença destas impurezas pode alterar as propriedades físico-químicas do biodiesel, hidrolisando os ésteres monoalquílico e dificultando seu armazenamento, além de promover a corrosão dos motores e produzir gases com maior teor de aldeídos e acroleína com a sua queima (Stojkoviĺ et al., 2014). Assim, para ser comercializado o biodiesel deve apresentar os parâmetros de qualidade conforme as especificações descritas nas regulamentações vigentes em cada país. A norma internacional (EN 14214) e a legislação brasileira (RANP 45/2014), determinam estes parâmetros de qualidades para que uma mistura de ésteres monoalquílicos possa ser classificada como biodiesel, como um teor mínimo de éster de $96,5 \%$ e concentrações de impurezas abaixo dos limites estabelecidos (Hayyan et al., 2010).

A técnica convencional de purificação do biodiesel por lavagem a úmido apresenta graves problemas ambientas, sendo que grandes volumes de efluentes são gerados (Shahbaz et al., 2013). Segundo HO et al. (2015), a purificação de $1 \mathrm{~L}$ de biodiesel gera aproximadamente $10 \mathrm{~L}$ de águas residuais. A saponificação dos ésteres é outro problema enfrentado, que reduz o rendimento do processo e requer a adição de mais água na lavagem (Ho et al., 2015). A secagem do biodiesel torna-se necessária para reduzir a umidade presente no combustível, além de outros processos para remover moléculas insolúveis em água, como os glicerídeos (mono-, di- e triglicerídeos) (Atadashi et al., 2011). Desta forma, o custo e complexidade dos processos envolvendo a purificação do biodiesel, tornam-se obstáculos para ampliar sua escala produtiva (Hayyan et al., 2010).

Neste contexto, os líquidos iônicos (LIôs), sais orgânicos compostos por íons, com temperatura de fusão inferior à de ebulição da água, ou seja, sais líquidos a temperatura ambiente, estão sendo amplamente estudados como solventes de lavagem na produção do biodiesel, constituindo uma opção ecologicamente mais correta. A capacidade em se manipular às propriedades físico-químicas (viscosidade, densidade, hidrofilicidade e solubilidade) através da composição do solvente, é uma característica importante dos LIô, tornando-os conhecidos como designer solventes (Francisco et al., 2013; Ohno et al., 2011; Pernak et al., 2005; Troter et al., 2016).

Os líquidos iônicos conhecidos como deep eutectic solventes, são formados pela mistura eutética de um aceitador de ligações de hidrogênio (HBA) e um, ou mais, doador de ligações de hidrogênio (HBD) (Figura 1), sendo que as fortes interações iônicas formadas justificam a diminuição da temperatura de fusão (Morrison et al., 2009; Zhang et al., 2012). Este tipo de LI tem inúmeras vantagens, como alta biodegradabilidade; baixa inflamabilidade e toxicidade; elevada estabilidade térmica e química; síntese simples; custo relativamente baixo; e pode ser recuperado e reciclados sem purificação (Florindo et al., 2014; Hayyan et al., 2013).

Em uma segunda etapa de purificação, o biodiesel 
HBA<smiles>C[N+](C)(C)CCO</smiles>

Cloreto de Colina

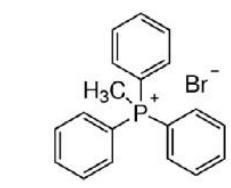

Brometo de Metil-trifenil-fosfônio
HBD
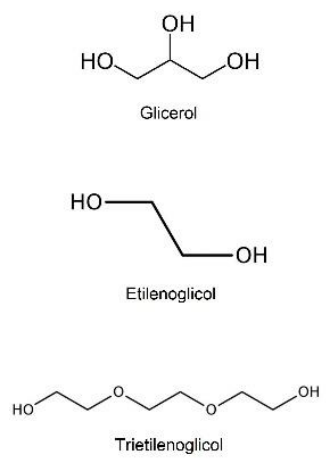

Figura 1 - Exemplos de aceitadores de ponte de hidrogênio (HBA) e doadores de ponte de hidrogênio (HBD) usados nas sínteses de líquidos iônicos.

A possibilidade em selecionar o HBA e o HBD, permite incluir a composição do líquido iônico, grupos funcionais específicos que iram influenciar nas interações solvente-soluto, como aqueles que apresentam ligações de hidrogênio (F-H, N-H e O-H). Assim, a alta polaridade dos LIôs permite a remoção das impurezas no biodiesel, como o glicerol, os ácidos graxos livres. Os LIôs também contribuem com a redução do teor de umidade e catalisador residual no biodiesel (Troter et al., 2016; Zhang et al., 2012).

$\mathrm{Na}$ literatura existem alguns trabalhos que empregaram líquidos iônicos como solvente na etapa de purificação do biodiesel. Abbott et al. (2007) utilizaram um LI composto por cloreto de colina e glicerol para purificar biodiesel de óleo de colza e óleo de soja, alcançando um teor final de glicerol livre de $0,02 \%$ e $0,06 \%$ respectivamente, ao misturar o solvente e o biodiesel em uma proporção molar de 1:1. Outros autores como, Shahbaz et al. (2013) e Hayyan et al. (2010), também purificaram biodiesel utilizando LI a base de cloreto de colina e glicerol. Shahbaz et al. (2011 a) também mostraram que o LI sintetizado a partir de cloreto de colina e glicerol reduz os teores de catalisador $\mathrm{KOH}$ (cerca de $98 \%$ ) e de água no biodiesel de óleo de palma, a valores abaixo dos limites exigidos pelas normas. Shahbaz et al. (2011 b) utilizaram na lavagem do biodiesel de óleo de palma um líquido iônico composto brometo de metil-trifenilfosfônio (HBA) e trietilenoglicol (HBD), removendo com eficiência o glicerol das amostras. No estudo da purificação do biodiesel do farelo de arroz, realizado por Niawanti et al. (2017), os autores obtiveram o aumento do teor de éster de $89,05 \%$ w até $96,60 \%$ w em quatro horas de lavagem, utilizando como solvente um LI composto por cloreto de colina e etilenoglicol, misturado ao biodiesel numa razão molar de 4:1 (LI:Biodiesel). Outros LIôs utilizando como HBD o etilenoglicol, foram utilizados para purificar biodiesel (Ho et al., 2015; Shahbaz et al., 2011 a; Shahbaz et al., 2010).

Considerando as diversas vias existentes na produção dos biocombustíveis, a separação, purificação e transformação da biomassa, correspondem por $60 \%-80 \%$ do custo do processo, representando um dos principais limitantes para o aumento da escala produtiva (Atadashi et al., 2011). Desta forma, pesquisadores apontam que um processo simples e eficiente de purificação para o biodiesel é imprescindível para redução dos custos produtivos, a fim de torna-lo competitivo no mercado energético (Sharma et al., 2008).

Este trabalho tem como objetivo desenvolver um novo LI empregado a purificação do biodiesel de soja, avaliando a sua eficiência no aumento do teor de éster por meio da remoção de impurezas não solúveis em água, para que o biocombustível purificado apresente conformidade com o a RANP 45/2014. Os treze solventes avaliados são sintetizados com uma mistura eutética simples de compostos biodegradáveis, não tóxicos, com baixo custo e alta disponibilidade (cloreto de colina, glicerol, etilenoglicol e trietilenoglicol), sem gerar subprodutos indesejáveis, em condições brandas de temperatura. Deve-se salientar que não foram encontrados relatos na literatura do uso de líquidos iônicos compostos por cloreto de colina e trietilenoglicol para purificar biodiesel.

Os deep eutectic solventes, são líquidos iônicos que podem ser desenvolvidos (designer solventes) para apresentar novas propriedades, ou promover o aumento da sua eficiência, de acordo com a composição selecionada. Assim, avaliar a influência da combinação de novos materiais com viabilidade econômica e ambiental, como o trietilenoglicol, sobre a eficiência da purificação do biodiesel, é fundamental para que a técnica possa ser integrada em processos de maior escala.

\section{MATERIAIS E MÉTODOS}

\subsection{Reagentes}

Na reação de transesterificação foram utilizados óleo de soja comercial, hidróxido de potássio $85 \%$ de pureza (Ahphatec) e metanol 99,8\% (Anidrol). Para a síntese dos líquidos iônicos foram usados cloreto de colina Purex $98 \%$ (Inlab), glicerol 99,5\% (Synth), etilenoglicol 99\% (Synth), trietilenoglicol 98\% (Dinâmica).

As análises realizadas em cromatógrafo a gás utilizaram de n-heptano grau HPLC de pureza (Honeywell) e heptadecanoato de metila 99\% (Sigma-Aldrich) como padrão interno.

\subsection{Reação de transesterificação}

Os ésteres metílicos foram produzidos por meio da reação de transesterificação do óleo de soja comercial por rota metílica e catálise básica e homogênea (Shahbaz et al., 2011 b). O metanol foi utilizado em excesso, em uma razão molar de mistura de 1:10 em relação ao óleo de soja, sendo previamente preparada uma solução metanólica com o catalisador hidróxido de potássio ( $2 \%$ w óleo). O óleo previamente aquecido foi misturado com a solução metanólica em um sistema de refluxo para o metanol, com agitação constante de $400 \mathrm{rpm}$ e temperatura de $68^{\circ} \mathrm{C}$, ao longo de 1 hora.

Ao término do tempo de reação, o volume foi transferido para um funil de decantação e após 24 horas se obteve a separação espontânea das fases do biodiesel bruto 
(superior) e do glicerol (inferior). $\mathrm{O}$ biodiesel bruto foi lavado em 6 etapas com água destilada a $60^{\circ} \mathrm{C}$, em cada etapa foram utilizados $250 \mathrm{ml}$ de água para cerca de $250 \mathrm{ml}$ de biodiesel bruto. Após a lavagem, o biodiesel foi seco em estufa por 3 horas a $105^{\circ} \mathrm{C}$ e armazenado em frasco âmbar. $\mathrm{Na}$ avaliação da eficiência dos líquidos iônicos foi utilizado o biodiesel pré-purificado (submetido a etapa de lavagem via úmida), uma vez que sua concentração em termos de ésteres era inferior aos padrões exigidos. A análise de eficiência de purificação nesta etapa foi baseada na concentração dos ésteres. A partir da análise dos resultados preliminares, foi escolhido um líquido iônico e realizado outros testes empregados biodiesel bruto (sem pré-tratamento por via úmida).

\subsection{Síntese dos líquidos iônicos}

O cloreto de colina (HBA) foi misturado ao glicerol, etilenoglicol e trietilenoglicol (HBDôs) conforme as razões molares apresentadas na Tabela 1 . A reação de síntese ocorreu a $80{ }^{\circ} \mathrm{C}$ com agitação constante de $300 \mathrm{rpm}$, ao longo de 1 hora em um sistema fechado, para evitar contaminações com o ar atmosférico (Ho et al., 2015).

\subsection{Lavagem do biodiesel}

O biodiesel pré-purificado foi submetido a purificação empregando líquidos iônicos como solvente. Os líquidos iônicos (Tabela 1) foram misturados ao biodiesel em quatro razões molares R1, R2, R3 e R4, respectivamente, 0.5:1, 1:1, 1.5:1 e 2:1 (LI:Biodiesel). As misturas foram agitadas a $200 \mathrm{rpm}$ em uma Incubadora com agitação orbital (New Lab), a temperatura de $30^{\circ} \mathrm{C}$, ao longo de 2 horas (Ho et al., 2015). Após o tempo de agitação, manteve-se o sistema em repouso por 2 horas, para que houvesse a separação do LI que é insolúvel na mistura de ésteres metílicos. O teor de éster do biodiesel purificado foi determinado em cromatógrafo a gás e o percentual de remoção obtido pela Equação 1.

Tabela 1 - Razões molares de mistura dos reagentes utilizados para a síntese dos líquidos iônicos.

\begin{tabular}{cc|c|c|c}
\multicolumn{5}{c}{ Razão Molar de Mistura } \\
LI & $\begin{array}{c}\text { HBA } \\
\text { Cloreto } \\
\text { de } \\
\text { Colina }\end{array}$ & Glicerol & Etilenoglicol & Trietilenoglicol \\
L1 & 1 & 2 & - & - \\
L2 & 1 & - & 2 & - \\
L3 & 1 & - & - & 2 \\
L4 & 1 & 1 & 1 & 1 \\
L5 & 1 & 1 & 1 & - \\
L6 & 1 & 2 & 1 & - \\
L7 & 1 & 1 & 2 & - \\
L8 & 1 & 1 & - & 1 \\
L9 & 1 & 2 & - & 1 \\
L10 & 1 & 1 & - & 2 \\
L11 & 1 & - & 1 & 1 \\
L12 & 1 & - & 2 & 1 \\
L13 & 1 & - & 1 & 2 \\
\hline
\end{tabular}

$\% \mathrm{R}=\frac{100-\%_{\mathrm{o}}-\left(100-\%_{\mathrm{f}}\right)}{100-\%_{\mathrm{o}}} .100$ em que $\% \mathrm{R}$ é a eficiência da remoção de impurezas na lavagem do biodiesel com LI (\%); \% é o teor de éster inicial no biodiesel não lavado (\%); e \% é o teor de éster na amostra de biodiesel lavado com LI (\%).

\subsection{Cinética de lavagem do biodiesel}

$\mathrm{O}$ efeito do tempo da lavagem do biodiesel com o líquido iônico que apresentou maior eficiência na remoção de impurezas, foi avaliado por meio da construção de uma curva cinética. $\mathrm{O}$ biodiesel utilizado foi sintetizado por meio da metodologia descrita (seção 2.2), alterando-se a razão molar de metanol para 1:3, limitando a produção de ésteres metílicos. A curva cinética foi construída variando-se o tempo de lavagem em 15, 30, 45, 60, 120 e 240 minutos, conforme metodologia descrita (seção2.4). O teor de éster de cada amostra, foi determinado por cromatografia a gás.

\subsection{Análises de cromatografia a gás}

A quantificação dos ésteres metílicos de ácidos graxos foi realizada empregando a técnica de cromatografia a gás (CG), sendo que foram utilizadas amostras com cerca de $0,3 \mathrm{~g}$, solubilizadas em $\mathrm{n}$-heptano, conforme a Normativa Europeia EN 14103. O padrão interno heptadecanoato de metila, com concentração conhecida, foi utilizado para determinar o teor de ésteres totais por normalização.

O cromatógrafo a gás PerkinElmer ï CLARUS 680, foi utilizado para a separação dos diferentes ésteres metílicos que compõem o biodiesel de soja, estando este equipado com sistema de injeção split e on-column (PSS), coluna capilar de sílica fundida de cianopropilpolisiloxano (Elite-WAX) nas dimensões de $30 \mathrm{~m}$ x $0,25 \mathrm{~mm}$ x $0,5 \mathrm{\varepsilon m}$ e detector por ionização de chama (FID). Nitrogênio foi usado como gás de arraste $\left(2 \mathrm{~mL} \cdot \mathrm{min}^{-1}\right)$, sendo injetados 2 عL (razão de split 1:50), estando o injetor a $250{ }^{\circ} \mathrm{C}$. A temperatura inicial do forno foi $160{ }^{\circ} \mathrm{C}$, alcançando $230^{\circ} \mathrm{C}$ a uma taxa de $5^{\circ} \mathrm{C}$. min ${ }^{-}$ ${ }^{1}$, permanecendo por $2 \mathrm{~min}$. O detector FID foi mantido a 250 ${ }^{\circ} \mathrm{C}$, sendo os gases de chama o hidrogênio $\left(45 \mathrm{~mL} \cdot \mathrm{min}^{-1}\right)$ e o ar sintético (450 mL. $\left.\mathrm{min}^{-1}\right)$.

O biodiesel de soja transesterificado via metanólica com catálise básica é composto majoritariamente por cinco ésteres de ácidos graxos, sendo eles o palmítico, esteárico, oleico, linoleico e linolênico (Almeida-Castilho et al., 2012; Navas et al., 2018; Wu et al., 2016). A Equação 2 adaptada de Visentainer e Franco (2006), permite determinar o teor de éster presentes em cada amostra de biodiesel injetada no cromatógrafo a gás (CG):

$\%_{\mathrm{x}}=\frac{\mathrm{M}_{\mathrm{p}}}{\mathrm{A}_{\mathrm{p}} \cdot \mathrm{M}_{\mathrm{o}}} \cdot 100$

em que, $\%_{\mathrm{x}}$ é o teor de éster na amostra (\%); $\mathrm{M}_{\mathrm{p}}$ é a massa de padrão interno presente na amostra $(\mathrm{mg}) ; \mathbf{M}_{\mathrm{o}}$ é a massa de biodiesel presente na amostra $(\mathrm{mg}) ; \mathrm{A}_{\mathrm{x}}$ é a soma das áreas relativa ao ésteres identificados no cromatograma ( $\varepsilon V . s) ; A_{p}$ é a área relativa ao padrão interno identificado no cromatograma ( $\varepsilon V . s)$.

\section{RESULTADOS E DISCUSSÃO}


O biodiesel bruto, não lavado, possui impurezas como glicerol, glicerídeos, umidade, metanol e catalisadores residuais, e a presença destas em meio a mistura de ésteres influencia consideravelmente no processo de purificação. Desta forma, a purificação do biodiesel bruto foi avaliada posterior a seleção do LI de maior eficiência (seção 3.3).

As amostras de biodiesel pré-purificadas utilizadas nos ensaios de remoção de impurezas, apresentavam um teor de éster de 90,86\% $\pm 0,6$ antes da lavagem com LI. Os autores Gonzalo et al. (2010), encontraram elevados teores de gliceróis na mistura de biodiesel após a lavagem a úmido, indicando baixa eficiência desta técnica na remoção de moléculas de baixa polaridade. Todos os solventes empregados foram capazes de reduzir as impurezas nas amostras de biodiesel de soja (Figura 2, Figura 3, Figura 4, e Figura 5), sendo necessária uma eficiência mínima de remoção de $61,71 \%$ para que o biodiesel alcance o teor mínimo de éster (96,5\%) requeridos pela RANP 45/2014.

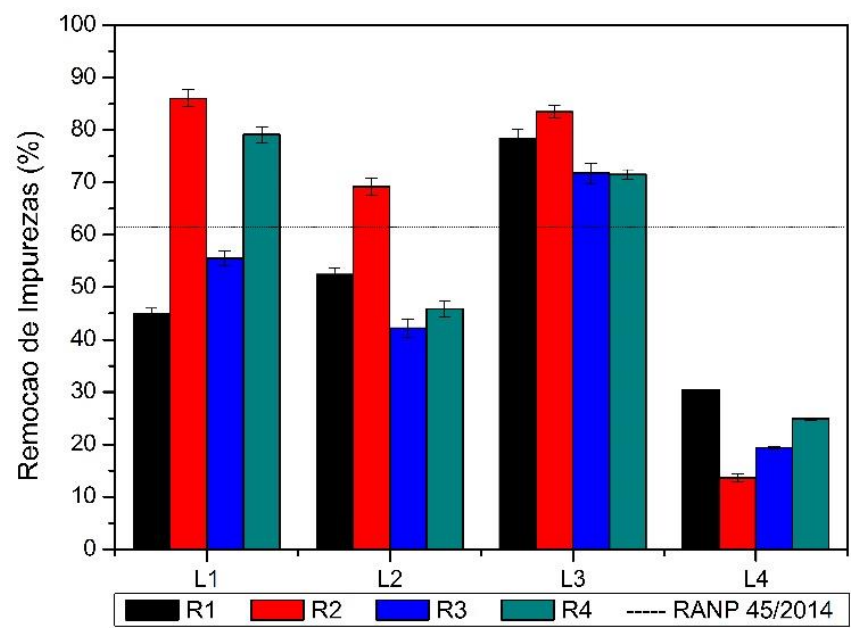

Figura 2 - Remoção de impurezas do biodiesel de soja pré-purificado, após a lavagem com os líquidos iônicos L1, L2, L3 e L4, com razões molares LI:Biodiesel R1, R2, R3 e R4.

Os solventes com apenas uma espécie de HBD (L1, L2 e L3), apresentaram uma eficiência satisfatória na purificação do biodiesel, com remoção máxima 86,03\% \$1,6 com o líquido iônico L1 quando misturado ao biodiesel na razão molar R2 (Figura 2). No entanto, a mistura eutética composta por um mol de cada espécie de HBD (L4), foi ineficiente na purificação, removendo no máximo $30,46 \% \pm 0,1$ das impurezas, valor que não atende ao determinado pela legislação (Figura 2). Os resultados obtidos na lavagem com L4, solvente ainda não avaliado em outros trabalhos, mostra que a combinação ternária dos HBDô, influenciou as interações intermoleculares, em especial as ligações de hidrogênio, reduzindo a solubilidade das impurezas no solvente.

Os autores Hayyan et al. (2010), Abbott et al (2007) e Shahbaz et al. (2011 a) utilizaram um líquido iônico com a mesma composição do L1, sendo observada a capacidade do solvente em reduzir a concentração de glicerídeos, glicerol, umidade e catalisador residual em amostras de biodiesel de óleo de palma. O solvente L2, assim como verificado por outros autores (Shahbaz et al., 2010; Shahbaz et al., 2011 a), é capaz de remover impurezas do biodiesel, no entanto não teve uma eficiência satisfatória em geral. A remoção máxima observada para L2 foi de $69,23 \% \pm 1,6$ em R2, resultado próximo ao encontrado por Niawantil et al. (2017), que removeu glicerídeos do biodiesel de óleo de farelo de arroz com o mesmo solvente.

Em todas as razões de mistura, o L3 foi satisfatório na remoção das impurezas do biodiesel, indicando que líquidos iônicos baseados em trietilenoglicol tem boa capacidade em solubilizar moléculas de baixa polaridade, como os glicerídeos. Shahbaz et al. (2011 b) utilizou solventes análogos ao L3, baseado no sal brometo de metiltrifenil-fosfônio e trietilenoglicol, identificando o mesmo comportamento, estes líquidos iônicos foram mais eficientes na purificação do biodiesel de óleo de palma, que aqueles baseados em glicerol ou etilenoglicol.

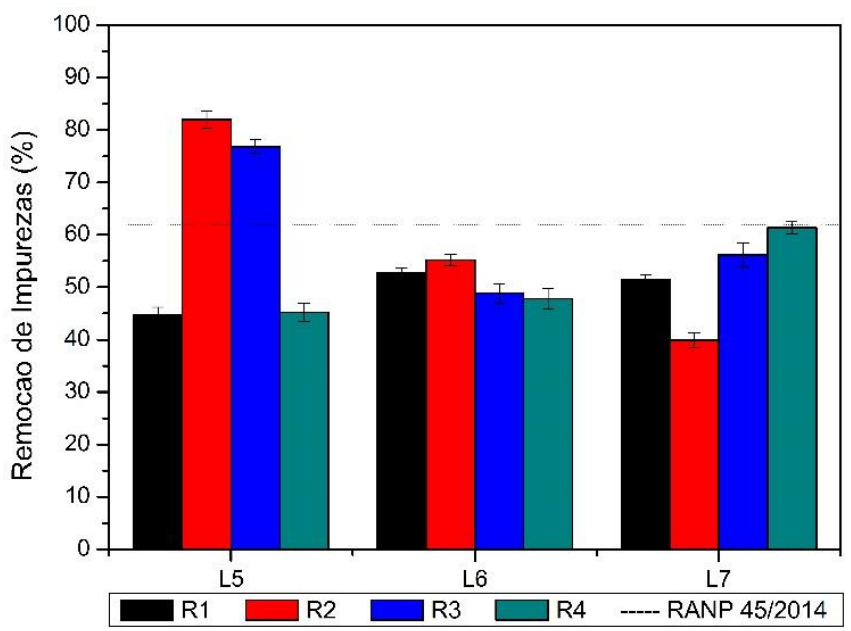

Figura 3 - Remoção de impurezas do biodiesel de soja pré-purificado, após a lavagem com os líquidos iônicos L5, L6 e L7, com razões molares LI:Biodiesel R1, R2, R3 e R4.

Os líquidos iônicos a base de cloreto de colina com HBD binários, glicerol e etilenoglicol (Figura 3), foram avaliados em outros trabalhos de purificação de biodiesel de óleo de palma (Ho et al., 2015). A maior remoção de impurezas ocorreu com o uso do líquido iônico L5, sendo igual a $81,98 \% \pm 1,7$ em razão de mistura $\mathrm{R} 2$, eficiência próxima a obtida na razão de mistura $\mathrm{R} 3$, com remoção de $76,84 \% \pm 1,2$. No entanto, a Figura 3 indica que o aumento da molaridade dos HBDô nos solventes L6 e L7, reduziu a eficiência da purificação, e após a lavagem as amostras de biodiesel não apresentaram conformidade com a RANP 45/2014. A adição de mols de etilenoglicol e, principalmente, glicerol, na síntese dos líquidos iônicos, altera as propriedades da mistura e influência nas atrações moleculares entre solvente-soluto, reduzindo a solubilidade das impurezas no líquido iônico, como apontado por Ho et al. (2015). 


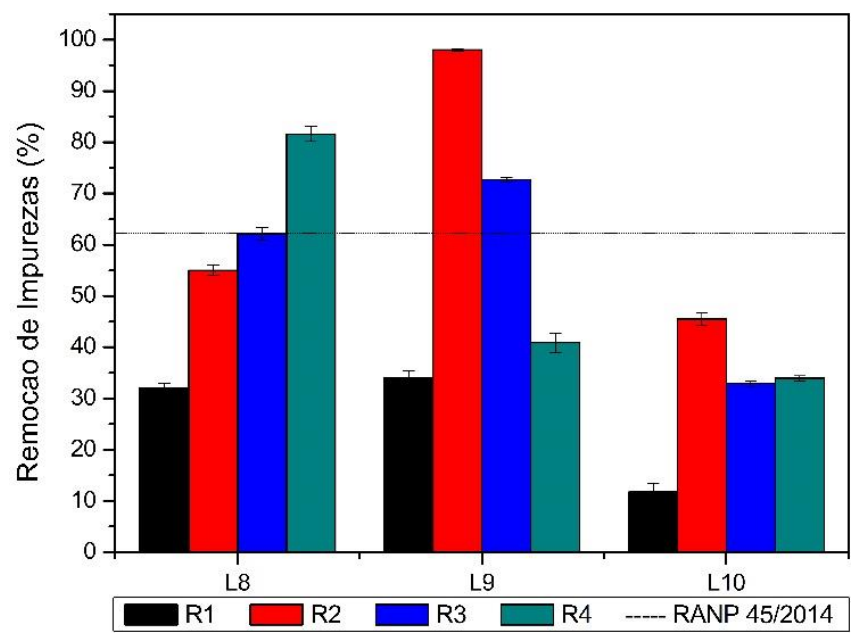

Figura 4 - Remoção de impurezas do biodiesel de soja pré-purificado, após a lavagem com os líquidos iônicos L8, L9 e L10, com razões molares LI:Biodiesel R1, R2, R3 e R4.

A combinação dos HBDô glicerol e trietilenoglicol, para a formação de novos líquidos iônicos, permitiu uma remoção de até $98,05 \% \pm 0,3$ ao ser utilizado o solvente L9 com razão molar de mistura LI:Biodiesel em R2 (Figura 4). Com o uso do LI L9, composto por cloreto de colina, glicerol e trietilenoglicol em razão molar de 1:2:1, obteve-se a maior eficiência de remoção de impurezas entre todos os solventes avaliados na lavagem do biodiesel de soja pré-purificado. As intensas interações intermoleculares (ligações de hidrogênio) estabelecidas entre os componentes do líquido iônico L9, atribuem ao solvente regiões moleculares de baixa polaridade, promovendo a solubilização eficiente das impurezas não removidas na lavagem a úmido. Resultados satisfatórios também foram obtidos com a lavagem usando o solvente L8, com remoção de até $81,60 \% \pm 1,5$, no entanto o aumento da molaridade de trietilenoglicol alterou a solubilidade das impurezas no solvente L10, e após a lavagem as amostras de biodiesel não apresentaram conformidade com a RANP 45/2014 (Figura 4).

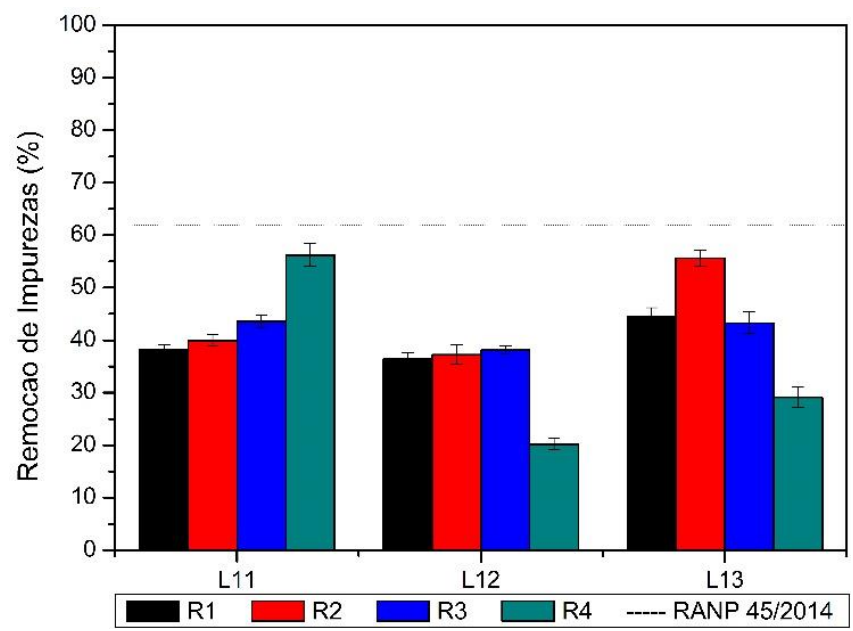

Figura 5 - Remoção de impurezas do biodiesel de soja pré-purificado, após a lavagem com os líquidos iônicos L11, L12 e L13, com razões molares LI:Biodiesel R1, R2, R3 e R4.
As purificações com L11, L12 e L13, foram ineficientes em todas as razões molares de LI:Biodiesel, sendo que as amostras não alcançaram o teor mínimo de éster determinado pela RANP 45/2014 (Figura 5). A combinação de etilenoglicol e trietilenoglicol, como HBDô para a síntese de líquidos iônicos, não atribuiu aos solventes a capacidade de solubilizar as impurezas do biodiesel de soja pré-tratado. Isto ocorre, pois as interações moleculares formadas entre o HBA e os HBDô, influenciam na polaridade do solvente, alterando as interações solvente-soluto em processos de extração líquido-líquido. Com maior eficiência de remoção de impurezas, o líquido iônico L9 foi utilizado nas demais análises, em uma razão R2 de mistura com o biodiesel.

\subsection{Efeito do tempo de lavagem}

Foi utilizado um biodiesel pré-purificado com menor concentração de ésteres para garantir que as amostras não atingissem rapidamente o equilíbrio.

Nas extrações líquido-líquido, o tempo é um parâmetro relevante que deve ser considerado no projeto de equipamentos para esta finalidade. $\mathrm{O}$ efeito do tempo de lavagem do biodiesel com o líquido iônico L9 (cloreto de colina, glicerol e trietilenoglicol) é apresentado na Figura 6, observa-se que o teor de éster aumentou em 8,05\% em 240 min de extração.

O aumento do teor de ésteres na lavagem do biodiesel, ocorre devido a remoção das impurezas diluídas na mistura, sendo esse fenômeno de transferência de massa favorecido pelo aumento do tempo de contato entre as moléculas de soluto-solvente. Niawanti et al. (2017) observaram um perfil cinético semelhante em seu trabalho de purificação de biodiesel de óleo de farelo de arroz, em que o teor de éster passa a ter uma variação pouco significante a partir de 60 min. A Figura 6 indica que o tempo de 60 minutos é adequado para que a lavagem do biodiesel ocorra de maneira satisfatória, removendo parcela considerável das impurezas.

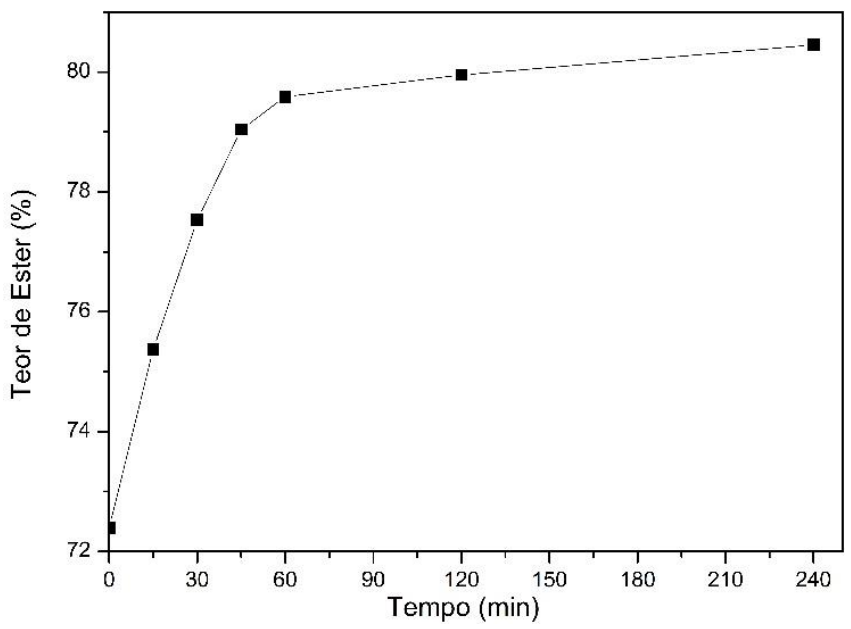

Figura 6 - Curva cinética de lavagem do biodiesel de soja. Lavado com líquido iônico L9 e razão molar de LI: Biodiesel = 1: 1. Os tempos de agitação foram de 0, 15, 30, 45, 60, 120 e 240 minutos.

\subsection{Purificação de biodiesel bruto}

O biodiesel bruto foi purificado com o líquido iônico L9, mostrando capacidade de reuso mesmo sem 
purificação (Tabela 2). Após a lavagem de 60 minutos com L9 puro, as impurezas solúveis no líquido iônico foram extraídas e o teor de éster alcançou 98,3\%, resultado que está em conformidade com a legislação (mín. 96,5\%). Como observado, após a primeira lavagem, o L9 pode ser novamente utilizado, no entanto o solvente fica saturado pelas moléculas de impurezas e perde eficiência de remoção com o $2^{\circ}$ reuso.

Tabela 2 - Lavagem do biodiesel bruto com o solvente L9 puro e reutilizado.

\begin{tabular}{|c|c|c|c|c|}
\hline L9 & Puro & $\mathbf{1}^{\mathbf{0}}$ Reuso & $\mathbf{2}^{\mathbf{0}}$ Reuso & $\mathbf{3}^{\mathbf{0}}$ Reuso \\
\hline $\begin{array}{c}\text { Teor de } \\
\text { Éster } \\
\text { (\%) }\end{array}$ & 98,3 & 97,7 & 93,7 & 93,8 \\
\hline
\end{tabular}

Entre as impurezas diluídas no biodiesel bruto, estão os glicerídeos, glicerol, água, metanol e catalisador residual $(\mathrm{KOH})$. As interações moleculares entre soluto (impurezas) e o solvente (LI L9) ocorrem devido a alta polaridade do LI, que forma ligações de hidrogênio com o grupo hidroxila $\left(\mathrm{OH}^{-}\right)$presente nas moléculas extraídas (Ho et al., 2015; Shahbaz et al., 2011 a). Assim, as ligações de hidrogênio estabelecidas entre as impurezas e o LI, tem caráter determinante na eficiência da purificação do biodiesel bruto.

Mesmo em escala laboratorial, o uso de líquidos iônicos na purificação do biodiesel de óleo de soja, mostrou ser uma alternativa de potencial para substituir técnicas tradicionais, como a lavagem a úmido. O método de purificação de biodiesel utilizando o LI L9 representa uma economia de até $83 \%$ do volume de solvente comparado ao uso de água em métodos tradicionais, sendo necessários cerca de 0,3 L de LI para purificar $1 \mathrm{~L}$ de biodiesel, além de significar uma otimização considerável do processo, que tem suas etapas simplificadas, tornando-o um método mais rápido e barato.

\section{CONCLUSÃO}

A composição dos LIô, influenciou a polaridade dos solventes estudados, afetando diretamente na eficiência da purificação do biodiesel pré-purificado. No entanto, a combinação de cloreto de colina, glicerol e trietilenoglicol, estabeleceu a formação de um novo solvente, o líquido iônico L9, que apresentou uma eficiência máxima de 98,05\% na remoção das impurezas insolúveis em água. As intensas forças intermoleculares entre soluto-solvente, devido a formação de ligações de hidrogênio, tornaram a lavagem do biodiesel com L9 altamente eficiente, apresentando um teor de ésteres em conformidade com a RANP 45/2014. O aumento do teor de ésteres foi proporcional ao tempo de lavagem, sendo atribuído ao tempo de 60 minutos o valor ótimo para a etapa de mistura LI:Biodiesel. O L9 também mostrou eficiência na lavagem do biodiesel bruto, que apresentava concentrações mais elevadas de impurezas, fornecendo resultados satisfatórios mesmo quando reutilizado sem purificação. Desta forma, o líquido iônico L9 mostra ter potencialidade de uso em processos reais, constituindo uma alternativa sustentável de solvente, em um método de purificação simples e eficiente.

\section{AGRADECIMENTOS}

Os autores agradecem ao $\mathrm{CNPq}$ pelo apoio financeiro. Este estudo foi financiado em parte pela Coordenação de Aperfeiçoamento de Pessoal de Nível Superior - Brasil (CAPES) - Código Financeiro $817693 / 2015$.

\section{REFERÊNCIAS}

ABBOTT, A. P.; CULLIS, P. M.; GIBSON, M. J.; HARRIS, R. C.; RAVEN, E. Extraction of glycerol from biodiesel into a eutectic based ionic liquid. Green Chemistry, v. 9, n. 8, p. 868-872, 2007.

ATADASHI, I. M.; AROUA, M. K.; AZIZ, A. A. Biodiesel separation and purification: A review. Renewable Energy, v. 36, p. 437-443, 2011.

BANKOVILïİ ILIĹ, I. B.; MILADINOVIĹ, M. R.; STAMENKOVIL, O. S.; VELJKOVIĹ, V. B. Application of nano CaÖ̈ based catalysts in biodiesel synthesis. Renewable and Sustainable Energy Reviews, v. 72, p. 746-760, 2017.

CASTILHO-ALMEIDA, E. W.; SANTOS, E. F.; MIRANDA, A. M.; JORIO, A.; FERREIRA, E. H. M.; ACHETE, C. A.; ARMOND, R. A. S. Z.; ANCONI, C. P. A.; ALMEIDA, W. B. Estudo teórico e experimental de espectros infravermelho de ésteres de ácido graxo presentes na composição do biodiesel de soja. Química Nova, v. 35, n. 9, p. 1752-1757, 2012.

FLORINDO, C.; OLIVEIRA, F. S.; REBELO, L. P; N.; FERNANDES, A. M.; MARRUCHO, I. M. Insights into the Synthesis and Properties of Deep Eutectic Solvents Based on Cholinium Chloride and Carboxylic Acids. ACS Sustainable Chemistry \& Engineering, v. 2, n. 10, p. 2416-2425, 2014.

FRANCISCO, M.; VAN DEN BRUINHORST, A.; KROON, M. C. Low-Transition-Temperature Mixtures (LTTMs): A New Generation of Designer Solvents. Angewandte Chemie International Edition, v. 52, n. 11, p. 3074-3085, 2013.

GONZALO, A.; GARCÍA, M.; SNCHEZ, J. L.; ARAUZO, J.; PEA, J. N. Water cleaning of biodiesel. Effect of catalyst concentration, water amount, and washing temperature on biodiesel obtained from rapeseed oil and used oil. Ind. Eng. Chem. Res, v. 49, n. 9, p. 4436-4443, 2010.

HAYYAN, M.; ALI, M.; HAYYAN, A.; AL-SAADI, M. A.; ALNASHEF, I. M.; MIRGHANI, M. E. S.; SAHEEDC, O. K. Are deep eutectic solvents benign or toxic?. Chemosphere, v. 90, n. 7, p. 2193-2195, 2013.

HAYYAN, M.; MJALLI, F. S.; HASHIM, M. A.; ALNASHEF, I. M. A novel technique for separating glycerine from palm oil-based biodiesel using ionic liquids. Fuel Processing Technology, v. 91, n. 1, p. 116-120, 2010

HO, K. C.; SHAHBAZ, K.; RASHMI, W.; MJALLI, F. S.; HASHIM, M. A.; ALNASHEF, I. M. Removal of glycerol from palm oil-based biodiesel using new ionic liquids analogues. Journal of Engineering Science and Technology, v. 10, n. 1, p. 98-111, 2015. 
ISHAK, Z. I.; SAIRI, N. A.; ALIAS, Y.; AROUA, M. K. T.; YUSOFF, R. A review of ionic liquids as catalysts for transesterification reactions of biodiesel and glycerol carbonate production. Catalysis Reviews, v. 59, n. 1, p. 44-93, 2017.

KAYODE, B.; HART, A. An overview of transesterification methods for producing biodiesel from waste vegetable oils. Biofuels, v. 10, n. 3, p. 419-437, 2019.

MARTIN, M.; SVENSSON, N.; EKLUND, M.; FONSECA, J. Production synergies in the current biofuel industry: opportunities for development. Biofuels, v. 3, n. 5, p. 545-554, 2012.

MORRISON, H. G.; SUN, C. C.; NEERVANNAN, S. Characterization of thermal behavior of deep eutectic solvents and their potential as drug solubilization vehicles. International Journal of Pharmaceutics, v. 378, n. 1-2, p. 136-139, 2009.

NAVAS, M. B.; LICK, I. D.; BOLLA, P. A.; CASELLA, M. L.;, RUGGERA, J. F. Transesterification of soybean and castor oil with methanol and butanol using heterogeneous basic catalysts to obtain biodiesel. Chemical Engineering Science, v. 187, p. 444-454, 2018.

NIAWANTIL, H.; ZULLAIKAHL, $\quad$ S.; RACHIMOELLAHL, M. Purification of Biodiesel by Choline Chloride Based Deep Eutectic Solvent. AIP Conference Proceedings, v. 1840, n. 1, 2017.

OHNO, H.; YOSHIZAWA, M.; MIZUMO, T. Electrochemical Aspects of Ionic Liquids. $2^{\mathrm{a}}$ ed., USA: John Wiley \& Sons, Inc., 2011.

PATEL, N. K.; SHAH, S. N. Food, Energy, and Water: The Chemistry Connection. Capítulo 11, USA: Elsevier, 2015.

PERNAK, J.; KUBIS-FEDER, J.; RAMANI, A.; ROBERTSON, J.; CIENIECKA-ROSL, A.; SEDDON, K. R. Synthesis, anti-microbial activities and anti-electrostatic properties of phosphoniumbased ionic liquids. Green Chemistry, v. 7, n. 12, p. 855-862, 2005.

SHAHBAZ, K.; MJALLI, F. S.; HASHIM, M. A.; ALNASHEF, I. M. Elimination of All Free Glycerol and Reduction of Total Glycerol from Palm Oil-Based Biodiesel Using Non-Glycerol Based Deep Eutectic Solvents. Separation Science and Technology, v. 48, p. 1184-1193, 2013.

SHAHBAZ, K.; MJALLI, F. S.; HASHIM, M. A.; ALNASHEF, I. M. Eutectic solvents for the removal of residual palm oil-based biodiesel catalyst. Separation and Purification Technology, v. 81, n. 2, p. 216-222, 2011 a

SHAHBAZ, K.; MJALLI, F. S.; HASHIM, M. A.; ALNASHEF, I. M. Using Deep Eutectic Solvents Based on Methyl Triphenyl Phosphunium Bromide for the Removal of Glycerol from Palm-Oil-Based Biodiesel. Energy \& Fuels, v. 25, n. 6, p. 2671-2678, $2011 \mathrm{~b}$.

SHAHBAZ, K.; MJALLI, F. S.; HASHIM, M. A.; ALNASHEF, I. M. Using Deep Eutectic Solvents for the Removal of Glycerol from Palm Oil-Based Biodiesel. Journal of Applied Sciences, v. 10, n. 24, p. 3349-3354, 2010.

SHARMA, Y. C.; SINGH, B.; UPADHYAY, S. N. Advancements in development and characterization of biodiesel: A review. Fuel, v. 87, n. 12, p. 23552373, 2008.

STOJKOVIĹ, I. J.; STAMENKOVIĹ, O. S.; POVRENOVIĹ, D. S.; VELJKOVIĹ, V. B. Puriýcation technologies for crude biodiesel obtained by alkali-catalyzed transesteriýcation. Renewable and Sustainable Energy Reviews, v. 32, n. 1-15, 2014.

TAHER, H.; NASHEF, E.; ANVAR, N.; AL-ZUHAIR, S. Enzymatic production of biodiesel from waste oil in ionic liquid medium. Biofuels, v. 10, n. 4, p. 463-472, 2019.

TROTER, D. Z.; TODOROVIĹ, Z. B.; nOKIĹSTOJANOVIĹ, D. R.; STAMENKOVIĹ, O. S,; VELJKOVIĹ, V. B. Application of ionic liquids and deep eutectic solventes in biodiesel production: A review. Renewable and Sustainable Energy Reviews, v. 61, p. 473-500, 2016.

VISENTAINER, J. V.; FRANCO, M. R. B. Ácidos Graxos em Óleos e Gorduras: identificação e quantificação. Brazil: Varela, 2006.

WU, L.; WEI, T.; TONG, Z.; ZOU, Y.; LIN, Z.; SUN, J. Bentonite-enhanced biodiesel production by $\mathrm{NaOH}-$ catalyzed transesterification of soybean oil with metanol. Fuel Processing Technology, v. 144, p. 334-340, 2016.

ZHANG, Q.; VIGIER, K. D. O.; ROYER, S.; JÉRÔME, F. Deep eutectic solventes: syntheses, properties and applications. Chemical Society Reviews, v. 41, n. 21, p. 7108-7146, 2012. 